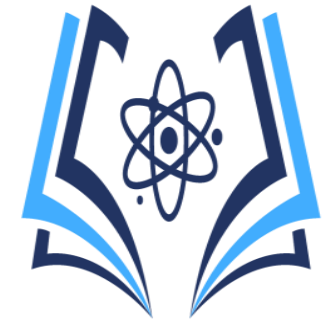

Martha Quiroz Figueroa ${ }^{1}$ Mercedes Lucas Choez ${ }^{2}$

\section{Rol de la enfermería en pacientes con complicaciones postoperatorias cardiovasculares}

\author{
Nursing role in patients with postoperative cardiovascular \\ complications
}

\section{Rol de la enfermería en pacientes con complicaciones}

\author{
${ }^{1}$ Universidad Estatal del Sur de Manabí, martha.quiroz@unesum.edu.ec, https://orcid.org/0000-0002- \\ 0959-0591 \\ ${ }^{2}$ Universidad Estatal del Sur de Manabí, https://orcid.org/0000-0003-2001-7611
}

Contacto: martha.quiroz@unesum.edu.ec

Recibido: 29-09-2019

Aprobado: 14-12-2019

\begin{abstract}
Resumen
El rol de enfermería en pacientes con complicaciones posquirúrgicas cardiovasculares es de gran importancia porque previene aquella eventualidad que ocurre en el curso previsto de un procedimiento quirúrgico, pero con el respectivo cuidado enfermero se les brinda una atención especializada en conjunto con el equipo multidisciplinario. El objetivo de este estudio fue determinar el rol de enfermería en pacientes con complicaciones cardiovasculares posoperatorias. Se realizó una revisión bibliográfica, en las diferentes bases de datos de carácter internacional y nacional; tales como Scielo, Cochrane, Bvs y Revicardiologia de artículos originales desde el 2015 al 2020, se encontraron más de 100 artículos relacionados al tema de los cuales se escogieron 24 artículos se obtuvo como resultado de eta investigación que el rol que desempeña la enfermera es el rol investigativo y el rol asistencial en lo que respecta las complicaciones en las que desempeñaron su rol fueron Taquicardias supraventriculares, paro cardiorrespiratorio y crisis hipertensiva postoperatoria ya que son las que mayor relevancia poseen. Conclusión el rol de la enfermería en el periodo postquirúrgico, es fundamental para que el paciente recupere sus funciones vitales que por efectos de la anestesia o por la propia cirugía están limitadas, en este periodo es fundamental garantizar la seguridad del paciente, aplicando cuidados de enfermería especializados, planificados e individualizados que permitan dar una atención integral y segura al paciente.
\end{abstract}

Palabras Claves: Atención de enfermería, Complicaciones, Complicaciones intraoperatorias.

\begin{abstract}
The nursing role in patients with post-surgical cardiovascular complications is of great importance because it prevents that eventuality that occurs during the planned course of a surgical procedure, but with the respective nursing care, specialized care is provided in conjunction with the multidisciplinary team. The objective of this study was to determine the role of nursing in patients with postoperative cardiovascular complications. A bibliographic review was carried out in the different international and national databases; such as Scielo, Cochrane, Bvs and Revicardiologia of original articles from 2015 to 2020, more than 100 articles related to the topic were found, of which 24 articles were chosen, as a result of this research, the role of the nurse is the The investigative role and the care role with regard to the complications in which they played their role were arrhythmias and arterial hypertension, since they are the ones with the greatest relevance. Conclusion the role of nursing in the postoperative period is essential for the patient to recover their vital functions that are limited by the effects of anesthesia or by the surgery itself, in this period it is essential to guarantee the safety of the patient, applying nursing care specialized, planned and individualized to provide comprehensive and safe patient care.
\end{abstract}


Key Words: Nursing care, Complications, Intraoperative complications.

\section{Introducción}

Las enfermeras, como profesionales que tratan de dar respuesta a las necesidades de la población, están sujetas a los cambios socio-demográficos, epidemiológicos y políticos que generan nuevas demandas a las que enfermería debe adaptarse por esto las nuevas necesidades sociales, sanitarias, políticas y económicas han conducido a la aparición de nuevos roles de enfermería que tratan de dar respuesta a la realidad social. El Proceso de Atención de Enfermería (P.A.E.) es la aplicación del método científico a la práctica asistencial de la enfermería. Este método permite a las enfermeras prestar cuidados de una forma racional, lógica y sistemática.

Conocer el cuidado como un concepto clave en la profesión de enfermería. Su significado y atribuciones han variado a lo largo del tiempo. Inicialmente, el cuidado estuvo relacionado con la supervivencia de la especie y fuertemente asociado al sexo femenino. En la actualidad, el cuidado enfermero no sólo comprende los conocimientos técnicos y teóricos sino las habilidades interpersonales entre otros.

El rol de enfermería en pacientes con complicaciones posquirúrgicas cardiovasculares es de gran importancia ya que ayuda a prevenir complicaciones aún más graves al paciente, con el respectivo cuidado enfermero se pueden disminuir riesgos futuros a los usuarios que padecen de estas enfermedades cardiovasculares y aquellos que tienen complicaciones más graves se les brinda una atención especializada en conjunto con el médico tratante.

Podemos decir que se define como complicación postoperatoria a aquella eventualidad que ocurre en el curso previsto de un procedimiento quirúrgico ya puede ser este en el postoperatorio inmediato o mediato con una respuesta local o sistémica que pueda retrasar la recuperación, poner en riesgo una función o hasta la vida.

Es importante destacar que las complicaciones cardiovasculares de una cirugía no cardíaca dependen de diversos factores: del tipo $y$ gravedad de las enfermedades previas que presentan los pacientes, del tipo de cirugía y del período postoperatorio. Es decir, cuando un enfermo comienza a respirar sin la ayuda de un respirador, inicia su movilización, siente dolor, etc. Por esto el período postoperatorio ha pasado a formar parte importante de las complicaciones cardiovasculares de la cirugía.

Entre las complicaciones más graves se encuentran: la muerte de origen cardíaco, infarto del miocardio, insuficiencia cardiaca, ya sea izquierda con edema pulmonar, o arritmias graves. Sin embargo, estas complicaciones son raras y hoy se da importancia como predictor de morbilidad a la isquemia miocárdica, porque es la complicación más frecuente en los pacientes sometidos a cirugía y a la vez su presencia constituye un factor de riesgo. Estas complicaciones se asocian con una mayor mortalidad hospitalaria, particularmente el paro cardiorrespiratorio y la insuficiencia cardiaca aguda; por ello, se deben tomar medidas preoperatorias y postoperatoria para la adecuada estratificación del riesgo y actuar oportunamente sobre los factores modificables.

\section{Materiales y métodos}

En la presente revisión bibliográfica, se consultaron diferentes bases de datos de carácter internacional y nacional; tales como Scielo, Cochrane, Bvs, Revicardiologia, Etc.

Diseño de estudio

En este proyecto se realizó revisiones sistemáticas que son investigaciones científicas de documentos originales desde el 2015 al 2020, donde se pudo evidenciar las principales complicaciones postquirúrgicas cardiovasculares; y el rol que desempeña en estos cuidados, además de los cuadros clínicos presentes en los pacientes y esto nos conlleva a planificar y ejecutar las acciones de enfermería en el posquirúrgico.

Población y muestra

Se encontraron más de 100 artículos relacionados al tema de investigación.

De los cuales se escogieron 20 artículos, los cuales aportaran al tema de investigación ya que poseen más acercamiento con dicho tema (complicaciones cardiovasculares posquirúrgicas)

Las palabras claves para esta revisión bibliográfica son:

- Complicaciones

cardiovasculares, hipertensión.

- Complicaciones cardiovasculares, arritmia.

- Complicaciones cardiovasculares, insuficiencia cardiaca aguda

- Complicaciones cardiovasculares, infarto al miocardio.

- Hipertensión arterial, complicación cardiovascular.

- Complicaciones hipertensión arterial.

cardiovasculares,

- Cirugía cardiaca, circulación extracorpórea, complicaciones postoperatorias. 


\section{HIGÍA DE LA SALUD}

- Cuidados Posoperatorios; Ansiedad; Depresión; Enfermería Perioperatoria; Cirugía Torácica; Complicaciones Posoperatorias.

- Cirugía oncológica, complicaciones cardiovasculares, paro cardiorrespiratorio, insuficiencia cardiaca aguda, resultados clínicos.

- Anestesia; Enfermedades Cardíacas.

- Enfermería. Cirugía cardiovascular. Arritmias Postoperatorias.

- Cardiopatías, congénitas, Arritmias, Cirugía cardíaca.

Técnicas utilizadas
ISSN 2773-7705

Periodo. Julio - Diciembre 2019

Vol. 1, Nro. 1, Publicado 2019-12-31

La técnica utilizada fue la recopilación bibliográfica, mediante el análisis exhaustivo de documentos fiables que presentan la información requerida, como, artículos científicos en revistas indexadas, publicaciones académicas etc. Con esta información se hace un compendio para el entendimiento general del tema.

\section{Resultados}

Se presentan los resultados de la revisión bibliográfica concerniente al rol de la en enfermera en complicaciones postoperatorias cardiovasculares, para lo cual se tomaron diversos estudios publicados desde el 2015 hasta el 2020 en revistas científicas indexadas regionales e internacionales.

\begin{tabular}{|c|c|c|c|c|c|}
\hline $\begin{array}{l}\text { Autores y } \\
\text { Nombre de } \\
\text { estudio }\end{array}$ & $\begin{array}{c}\text { Base de } \\
\text { datos }\end{array}$ & Año & Palabra clave & Complicaciones & $\begin{array}{c}\text { Rol de } \\
\text { enfermería }\end{array}$ \\
\hline $\begin{array}{l}\text { G Orlandova;L } \\
\text { Bello; D } \\
\text { Anchundia } \\
\text { Cirugía } \\
\text { cardíaca, } \\
\text { complicaciones } \\
\text { inmediatas post } \\
\text { operatorias. }\end{array}$ & Scielo & 2020 & $\begin{array}{l}\text { Complicaciones } \\
\text { cardiovasculare } \\
\text { s (insuficiencia } \\
\text { cardiaca aguda } \\
\text { intervención } \\
\text { quirúrgica) }\end{array}$ & $\begin{array}{l}\text { - } \text { Crisis } \\
\text { hipertensiva } \\
\text { - Insuficiencia } \\
\text { cardiaca aguda } \\
\text { - Arritmia }\end{array}$ & $\begin{array}{l}\text { Se aplicó el rol de } \\
\text { enfermería } \\
\text { investigativo ya } \\
\text { que el estudio fue } \\
\text { creado por un } \\
\text { grupo de } \\
\text { enfermeras. }\end{array}$ \\
\hline $\begin{array}{l}\text { A Alzate } \\
\text { Moctezuma ; B } \\
\text { Arce Bojórquez; } \\
\text { F Peraza Garay } \\
\text { Incidencia de } \\
\text { lesión } \\
\text { miocárdica } \\
\text { aguda } \\
\text { postoperatoria } \\
\text { en pacientes con } \\
\text { hipertensión } \\
\text { arterial } \\
\text { sistémica } \\
\text { sometidos a no } \\
\text { cirugía cardiaca }\end{array}$ & $\begin{array}{l}\text { Cochrane } \\
\text { biblioteca } \\
\text { virtual en } \\
\text { salud }\end{array}$ & 2018 & $\begin{array}{l}\text { Lesión } \\
\text { miocárdica } \\
\text { aguda } \\
\text { postoperatoria- } \\
\text { troponinas }\end{array}$ & $\begin{array}{l}\text { La lesión } \\
\text { miocárdica aguda } \\
\text { postoperatoria es } \\
\text { una de las } \\
\text { complicaciones } \\
\text { cardiovasculares } \\
\text { más frecuentes. }\end{array}$ & $\begin{array}{l}\text { No se aplicó } \\
\text { ningún rol de } \\
\text { enfermería }\end{array}$ \\
\hline $\begin{array}{l}\text { L. Hernández, } \\
\text { G. Reynoso } \\
\underline{\text { García. }} \\
\text { Cuidado de } \\
\text { enfermería } \\
\text { postoperación } \\
\text { de Trombo } \\
\text { endarterectomía } \\
\text { Pulmonar } \\
\text { Bilateral. } \\
\text { Estudio de caso } \\
\text { fundamentado }\end{array}$ & $\begin{array}{l}\text { Biblioteca } \\
\text { virtual en } \\
\text { salud } \\
\text { (BVS) } \\
\text { Scielo }\end{array}$ & 2019 & $\begin{array}{l}\text { Trombo } \\
\text { endarterectomía } \\
\text {; hipertensión } \\
\text { pulmonar; } \\
\text { síndrome de } \\
\text { Klinefelter; } \\
\text { cuidados } \\
\text { postoperatorios; } \\
\text { atención de } \\
\text { enfermería; } \\
\text { cuidados } \\
\text { críticos }\end{array}$ & $\begin{array}{l}\text { Las posibles } \\
\text { complicaciones } \\
\text { que puede } \\
\text { presentar son } \\
\text { alteración del } \\
\text { ritmo cardiaco, } \\
\text { insuficiencia } \\
\text { respiratoria } \\
\text { aguda y eventos } \\
\text { trombóticos } \\
\text { secundarios. }\end{array}$ & $\begin{array}{l}\text { Se aplico el rol de } \\
\text { enfermería } \\
\text { investigativo y el } \\
\text { asistencial. }\end{array}$ \\
\hline
\end{tabular}




\begin{tabular}{|c|c|c|c|c|c|}
\hline $\begin{array}{l}\text { en los principios } \\
\text { de Henderson }\end{array}$ & & & & & \\
\hline $\begin{array}{l}\text { Wai-Tat Wong, } \\
\text { Veronica KW } \\
\text { Lai, Yee Eot } \\
\text { Chee, } \\
\text { Anna Lee } \\
\text { Cuidado } \\
\text { cardiaco rápido } \\
\text { para pacientes } \\
\text { adultos } \\
\text { sometidos a } \\
\text { cirugía cardíaca }\end{array}$ & $\begin{array}{l}\text { Cochrane } \\
\text { Database of } \\
\text { Systematic } \\
\text { Reviews }\end{array}$ & 2016 & $\begin{array}{l}\text { Complicaciones } \\
\text { accidentes } \\
\text { cerebrovascular } \\
\text { es } \\
\text { Con } 17.000 \\
\text { resultados } \\
\text { debido al } \\
\text { acercamiento } \\
\text { con nuestro } \\
\text { tema principal } \\
\text { de PIS solo } \\
\text { usamos el } \\
\text { presente }\end{array}$ & \begin{tabular}{|l} 
- \\
Infarto al \\
miocardio \\
Accidentes \\
cerebrovasculare \\
$\mathrm{s}$
\end{tabular} & $\begin{array}{l}\text { No se aplicó } \\
\text { ningún rol de } \\
\text { enfermería. }\end{array}$ \\
\hline 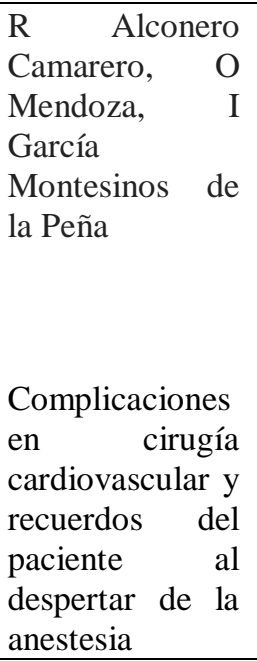 & Scielo & 2019 & $\begin{array}{l}\text { Procedimientos } \\
\text { quirúrgicos } \\
\text { cardiovasculare } \\
\text { s, } \\
\text { complicaciones } \\
\text { intraoperatorias, } \\
\text { complicaciones } \\
\text { postoperatorias, } \\
\text { dolor } \\
\text { postoperatorio, } \\
\text { cooperación del } \\
\text { paciente. }\end{array}$ & $\begin{array}{l}\text { Las } \\
\text { complicaciones } \\
\text { más habituales } \\
\text { arritmias. }\end{array}$ & $\begin{array}{l}\text { No se aplicó } \\
\text { ningún rol de } \\
\text { enfermería. }\end{array}$ \\
\hline 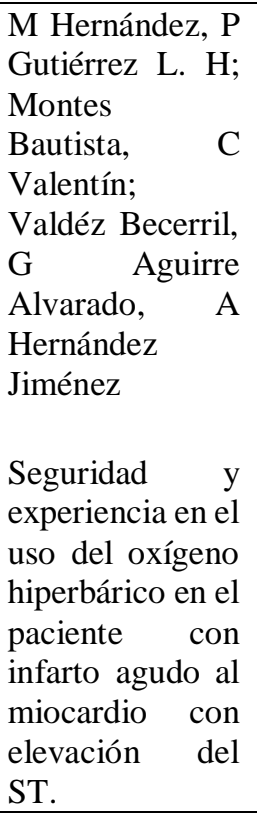 & Scielo & 2019 & $\begin{array}{l}\text { Complicaciones } \\
\text { cardiovasculare } \\
\text { s; infarto al } \\
\text { miocardio }\end{array}$ & $\begin{array}{l}\text { Complicaciones } \\
\text { infarto al miocardio }\end{array}$ & $\begin{array}{l}\text { No se aplicó } \\
\text { ningún rol de } \\
\text { enfermería. }\end{array}$ \\
\hline $\begin{array}{l}\text { Kahn, S R ; } \\
\text { Morrison, D R ; } \\
\text { Diendéré, G ; } \\
\text { Piché, A ; Filion, } \\
\text { K B ; Klil Drori, } \\
\text { A J ; Douketis, J } \\
\text { D ; Emed, J ; } \\
\text { Roussin, A ; }\end{array}$ & $\begin{array}{l}\text { Biblioteca } \\
\text { virtual de } \\
\text { salud BVS }\end{array}$ & 2018 & $\begin{array}{l}\text { Tromboembolis } \\
\text { mo pulmonar }\end{array}$ & $\begin{array}{l}\text { Tromboembolis } \\
\text { mo pulmonar }\end{array}$ & $\begin{array}{l}\text { No se aplicó } \\
\text { ningún rol de } \\
\text { enfermería. }\end{array}$ \\
\hline
\end{tabular}




\begin{tabular}{|c|c|c|c|c|c|}
\hline $\begin{array}{l}\text { Tagalakis, V ; } \\
\text { Morris, M ; } \\
\text { Geerts, William } \\
\text { Intervenciones } \\
\text { para la } \\
\text { implementación } \\
\text { de } \\
\text { tromboprofilaxi } \\
\text { s en pacientes } \\
\text { hospitalizados } \\
\text { con riesgo de } \\
\text { tromboembolis } \\
\text { mo venoso. }\end{array}$ & & & & & \\
\hline 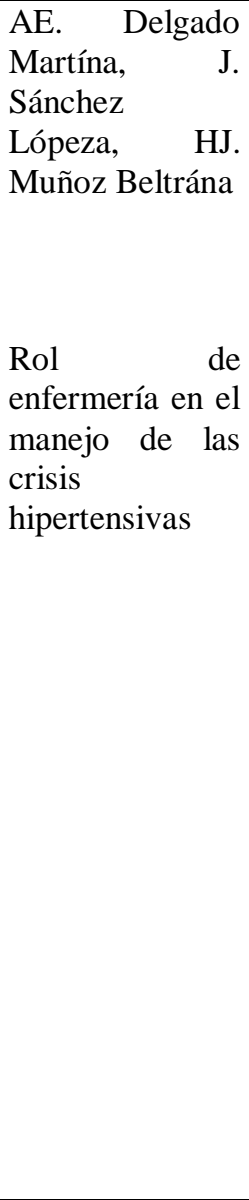 & $\begin{array}{l}\text { Cochrane } \\
\text { biblioteca } \\
\text { virtual en } \\
\text { salud }\end{array}$ & 2016 & $\begin{array}{l}\text { Crisis } \\
\text { hipertensivas }\end{array}$ & $\begin{array}{l}\text { Crisis hipertensivas } \\
\text { Arritmias } \\
\text { Taquicardia }\end{array}$ & $\begin{array}{l}\text { Se aplica el rol } \\
\text { asistencial de } \\
\text { enfermería,(ya que } \\
\text { mientras una crisis } \\
\text { hipertensiva está } \\
\text { pendiente del } \\
\text { paciente dando su } \\
\text { diurético en el } \\
\text { horario adecuado) } \\
\text { el rol } \\
\text { investigativo(debi } \\
\text { do a que fue } \\
\text { realizado dentro } \\
\text { de los autores por } \\
\text { un profesional de } \\
\text { enfermería),el rol } \\
\text { educativo(ya que } \\
\text { cuando ran } \\
\text { paciente tenga una } \\
\text { crisis hipertensiva } \\
\text { post operatoria, el } \\
\text { profesional de } \\
\text { enfermería se ve } \\
\text { en el puesto de } \\
\text { auto educar al } \\
\text { paciente a que el } \\
\text { mismo sepa el } \\
\text { horario correcto de } \\
\text { la toma de su } \\
\text { diurético). }\end{array}$ \\
\hline $\begin{array}{l}\text { S Burgués } \\
\text { Insuficiencia } \\
\text { cardíaca aguda }\end{array}$ & Scielo & 2017 & $\begin{array}{l}\text { Insuficiencia } \\
\text { cardíaca aguda } \\
\text { (ICA) }\end{array}$ & $\begin{array}{ll}\text { - } & \begin{array}{l}\text { Insuficiencia } \\
\text { cardiaca aguda } \\
\text { (ICA) }\end{array} \\
\text { - } & \text { Síndromes } \\
\text { coronarios } \\
\text { agudos. } \\
\text { - } \text { Hipertensión. } \\
\text { - Arritmias. } \\
\text { - } \text { Complicacione } \\
\text { s mecánicas } \\
\text { agudas. } \\
\text { Embolismo } \\
\text { pulmonar. }\end{array}$ & $\begin{array}{lr}\text { Dentro de } & \text { la } \\
\text { aplicación de los } \\
\text { roles } & \text { de } \\
\text { enfermería } & \\
\text { encontramos en } \\
\text { rol asistencias } \\
\text { debido a que } \\
\text { enfermería asiste } \\
\text { de manera directa } \\
\text { en el post } \\
\text { operatorio a } \\
\text { pacientes con } \\
\text { insuficiencia } \\
\text { cardiaca aguda, el } \\
\text { rol educativo ya } \\
\text { que enfermería }\end{array}$ \\
\hline
\end{tabular}




\begin{tabular}{|c|c|c|c|c|c|}
\hline & & & & & $\begin{array}{l}\text { educa a los } \\
\text { pacientes con } \\
\text { complicaciones } \\
\text { post quirúrgica } \\
\text { cardiovasculares } \\
\text { una vez que se } \\
\text { encuentran en su } \\
\text { etapa de } \\
\text { recuperación en el } \\
\text { potes operatorios, } \\
\text { para encaminar al } \\
\text { paciente a que su } \\
\text { salud se recupere y } \\
\text { por ende se dé su } \\
\text { alta, y el rol } \\
\text { investigativo } \\
\text { debido a que } \\
\text { enfermería } \\
\text { investiga el uso de } \\
\text { técnicas no } \\
\text { farmacológicas( } \\
\text { como } \\
\text { ultrafiltración) } \\
\text { para la } \\
\text { disminución de } \\
\text { dicha afección en } \\
\text { el caso de que los } \\
\text { pacientes r lo } \\
\text { requieran. }\end{array}$ \\
\hline 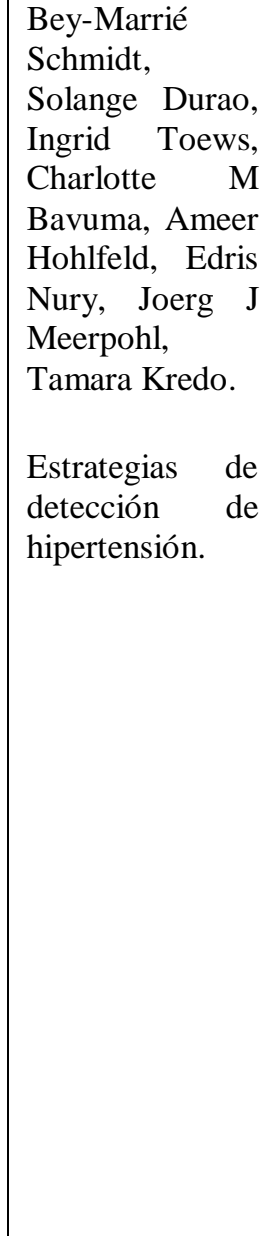 & Cochrane & 2020 & $\begin{array}{l}\text { Complicaciones } \\
\text { cardiovascular } \\
\text { hipertensión }\end{array}$ & $\begin{array}{l}\text { A largo plazo, la } \\
\text { hipertensión es un } \\
\text { factor de riesgo } \\
\text { importante para } \\
\text { eventos } \\
\text { cardiovasculares, } \\
\text { como enfermedades } \\
\text { cardíacas, } \\
\text { accidentes } \\
\text { cerebrovasculares e } \\
\text { insuficiencia renal, } \\
\text { así como } \\
\text { discapacidad yol y } \\
\text { mortalidad ( OMS } \\
\text { prematura ( OMa } \\
\text { 2013). Los factores } \\
\text { que aumentan el } \\
\text { riesgo de } \\
\text { hipertensión } \\
\text { incluyen factores } \\
\text { genéticos y de estilo } \\
\text { de vida, como el } \\
\text { consumo excesivo } \\
\text { de sal y grasas, la } \\
\text { inactividad física, el } \\
\text { consumo nocivo de } \\
\text { alcohol y el mal } \\
\text { manejo del estrés ( } \\
\text { OMS 2013 ). La } \\
\text { creciente evidencia } \\
\text { muestra que las }\end{array}$ & $\begin{array}{l}\text { No se aplicó } \\
\text { ningún rol de } \\
\text { enfermería. }\end{array}$ \\
\hline
\end{tabular}




\begin{tabular}{|c|c|c|c|c|c|}
\hline & & & & $\begin{array}{l}\text { personas más } \\
\text { jóvenes, como los } \\
\text { adolescentes, } \\
\text { también tienen } \\
\text { riesgo de } \\
\text { hipertensión debido } \\
\text { a estos factores de } \\
\text { estilo de vida poco } \\
\text { saludables ( Cheung } \\
2017 \text {; Kar 2015). }\end{array}$ & \\
\hline $\begin{array}{l}\text { Elena Buzzetti, } \\
\text { Maria } \\
\text { Kalafateli, } \\
\text { Douglas } \\
\text { Thorburn, Brian } \\
\mathrm{R} \text { Davidson, } \\
\text { Emmanuel } \\
\text { Tsochatzis, } \\
\text { Kurinchi Selvan } \\
\text { Gurusamy } \\
\text { Intervenciones } \\
\text { para la } \\
\text { hemocromatosis } \\
\text { hereditaria }\end{array}$ & Cochrane & 2020 & $\begin{array}{l}\text { Complicaciones } \\
\text { cardiovascular } \\
\text { arritmia }\end{array}$ & $\begin{array}{l}\text { Los síntomas } \\
\text { comunes } \\
\text { relacionados con la } \\
\text { hemocromatosis } \\
\text { hereditaria en el } \\
\text { momento del } \\
\text { diagnóstico son } \\
\text { mala salud general, } \\
\text { fatiga, malestar } \\
\text { general, diabetes y } \\
\text { artralgia } \\
\text { Pietrangelo 2004; } \\
\text { Allen 2008; van } \\
\text { Bokhoven 2011 ). } \\
\text { Las complicaciones } \\
\text { relacionadas con la } \\
\text { hemocromatosis } \\
\text { hereditaria incluyen } \\
\text { cirrosis hepática (y } \\
\text { complicaciones } \\
\text { relacionadas como } \\
\text { insuficiencia } \\
\text { hepática } \\
\text { carcinoma y } \\
\text { hepatocelular), } \\
\text { insuficiencia } \\
\text { cardíaca, arritmias } \\
\text { cardíacas, } \\
\text { impotencia, } \\
\text { diabetes, artritis y } \\
\text { pigmentación de la } \\
\text { piel ( Pietrangelo } \\
\text { 2004; Schmitt 2005 } \\
\text {; van Bokhoven } \\
\text { 2011 ; Bardou - } \\
\text { Jacquet 2014). }\end{array}$ & $\begin{array}{l}\text { No se aplicó } \\
\text { ningún rol de } \\
\text { enfermería. }\end{array}$ \\
\hline $\begin{array}{l}\text { Carratalá, José } \\
\text { Manuel; Díaz } \\
\text { Lobato2,3, } \\
\text { Salvador; } \\
\text { Llorens', Pere. } \\
\\
\text { Terapia de alto } \\
\text { flujo con } \\
\text { cánulas nasales } \\
\text { en pacientes con } \\
\text { insuficiencia } \\
\text { cardíaca aguda. }\end{array}$ & Scielo & 2018 & $\begin{array}{l}\text { Complicaciones } \\
\text { cardiovascular } \\
\text { insuficiencia } \\
\text { cardiaca aguda }\end{array}$ & $\begin{array}{l}\text { La gran mayoría de } \\
\text { pacientes con ICA } \\
\text { se presentan con } \\
\text { disnea13 y en casi el } \\
50 \% \text { asocian } \\
\text { hipoxemia, } \\
\text { hipercapnia, } \\
\text { acidosis o una } \\
\text { combinación de } \\
\text { estos14. En relación } \\
\text { con los diferentes } \\
\text { síndromes de ICA, } \\
\text { la IRA se asocia de } \\
\text { forma significativa }\end{array}$ & $\begin{array}{l}\text { No se aplicó } \\
\text { ningún rol de } \\
\text { enfermería. }\end{array}$ \\
\hline
\end{tabular}




\begin{tabular}{|c|c|c|c|c|c|}
\hline & & & & $\begin{array}{l}\text { en el edema de } \\
\text { pulmón } \\
\text { cardiogénico (EAP), } \\
\text { en el shock } \\
\text { cardiogénico y en la } \\
\text { insuficiencia } \\
\text { cardíaca crónica o } \\
\text { de novo, bien } \\
\text { aisladas o bien } \\
\text { asociadas a otras } \\
\text { enfermedades o } \\
\text { alteraciones } \\
\text { pulmonares } \\
\text { concomitantes } \\
\text { bien que actúan } \\
\text { como factores } \\
\text { precipitantes15 } \\
\text { (infecciones } \\
\text { respiratorias, } \\
\text { hiperreactividad } \\
\text { bronquial, } \\
\text { exacerbación de } \\
\text { enfermedad } \\
\text { pulmonar } \\
\text { obstructiva crónica - } \\
\text { EPOC-). }\end{array}$ & \\
\hline $\begin{array}{l}\text { Moreira, } \\
\text { Eduardo; Pintos, } \\
\text { Nury; Arteaga, } \\
\text { María De; } \\
\text { Negreira, } \\
\text { Sandra Torres; } \\
\text { Roza, Rita Da; } \\
\text { Albornoz, } \\
\text { Henry; Burghi, } \\
\text { Gastón. } \\
\text { Infarto agudo de } \\
\text { miocardio lejos } \\
\text { de los centros de } \\
\text { hemodinamia, } \\
\text { percepción de la } \\
\text { calidad de vida } \\
\text { postratamiento } \\
\text { con } \\
\text { fibrinolíticos y } \\
\text { factores } \\
\text { relacionados. }\end{array}$ & Scielo & 2019 & $\begin{array}{l}\text { Complicaciones } \\
\text { cardiovascular } \\
\text { infarto al } \\
\text { miocardio. }\end{array}$ & $\begin{array}{lr}\text { Se consideró } \\
\text { insuficiencia } \\
\text { cardíaca } \\
\text { cualquier } \quad \text { (IC) } \\
\text { hospitalario por este } \\
\text { síndrome } \\
\text { evidencia } \\
\text { fracción de eyección } \\
\text { del de } \\
\text { izquierdo ventrículo } \\
\text { disminuida. }\end{array}$ & $\begin{array}{l}\text { No se aplicó } \\
\text { ningún rol de } \\
\text { enfermería. }\end{array}$ \\
\hline $\begin{array}{lr}\text { Scott } & \mathrm{R} \\
\text { Garrison, } \\
\text { Michael } \\
\text { Kolber, } \\
\text { Christina } \\
\text { Korownyk, Rita } \\
\text { K McCracken, } \\
\text { Balraj S Heran, } \\
\text { G Michael } \\
\text { Allan. }\end{array}$ & Cochrane & 2017 & $\begin{array}{l}\text { Hipertensión } \\
\text { arterial } \\
\text { complicación } \\
\text { cardiovascular }\end{array}$ & $\begin{array}{l}\text { Las presiones más } \\
\text { altas aumentan el } \\
\text { riesgo de eventos } \\
\text { adversos para la } \\
\text { salud como } \\
\text { accidente } \\
\text { cerebrovascular, } \\
\text { ataque cardíaco, } \\
\text { insuficiencia } \\
\text { cardíaca y muerte. } \\
\text { Se ha demostrado } \\
\text { que reducir la PA }\end{array}$ & $\begin{array}{l}\text { No se aplicó } \\
\text { ningún rol de } \\
\text { enfermería. }\end{array}$ \\
\hline
\end{tabular}




\begin{tabular}{|c|c|c|c|c|c|}
\hline $\begin{array}{lr}\text { Objetivos } & \text { de } \\
\text { presión arterial } \\
\text { para } \\
\text { hipertensión en } \\
\text { adultos } \\
\text { mayores. }\end{array}$ & & & & $\begin{array}{l}\text { con medicamentos } \\
\text { reduce el riesgo de } \\
\text { estos eventos de } \\
\text { salud graves, pero se } \\
\text { desconoce el } \\
\text { objetivo óptimo de } \\
\text { PA cuando se trata a } \\
\text { adultos mayores. }\end{array}$ & \\
\hline $\begin{array}{lr}\text { James } & \text { M } \\
\text { Wright, Vijaya } \\
\text { M Musini y } \\
\text { Rupam Gill } \\
\\
\\
\\
\begin{array}{lr}\text { Fármacos de } \\
\text { primera línea } \\
\text { para la } \\
\text { hipertensión }\end{array}\end{array}$ & Cochrane & 2018 & $\begin{array}{l}\text { Complicaciones } \\
\text { cardiovasculare } \\
\text { s, hipertensión } \\
\text { arterial. }\end{array}$ & $\begin{array}{lrr}\text { No } & \text { presentan } \\
\text { signos ni } & \text { síntomas, } \\
\text { incluso si } & \text { las } \\
\text { lecturas de } & \text { presión } \\
\text { arterial son } & \text { muy } \\
\text { altas. Para } & \text { la } \\
\text { mayoría de } & \text { los } \\
\text { adultos } & \text { con } \\
\text { hipertensión } \\
\text { primaria o esencial, } \\
\text { no existe una causa } \\
\text { identificable para la } \\
\text { presión arterial alta. } \\
\text { Algunas personas } \\
\text { tienen presión } \\
\text { arterial alta, llamada } \\
\text { hipertensión } \\
\text { secundaria, causada } \\
\text { por afecciones } \\
\text { subyacentes como } \\
\text { tumores de la } \\
\text { glándula } \\
\text { suprarrenal, } \\
\text { problemas renales }\end{array}$ & $\begin{array}{l}\text { No se aplicó } \\
\text { ningún rol de } \\
\text { enfermería. }\end{array}$ \\
\hline $\begin{array}{l}\text { Hélen Francine } \\
\text { Rodrigues } \\
\text { Rejane Kiyoma } \\
\text { Furuya } \\
\text { Rosana } \\
\text { Aparecida } \\
\text { Spadoti Dantas } \\
\text { Alfredo José } \\
\text { Rodrigues } \\
\text { Carina } \\
\text { Aparecida } \\
\text { Marosti } \\
\text { Dessotte3 } \\
\text { Asociaciones de } \\
\text { los síntomas de } \\
\text { ansiedad y } \\
\text { depresión } \\
\text { preoperatorios } \\
\text { con complicaciones } \\
\text { postoperatorias } \\
\text { de cirugías } \\
\text { cardiacas }\end{array}$ & Scielo & 2018 & $\begin{array}{l}\text { Cuidados } \\
\text { Posoperatorios; } \\
\text { Ansiedad; } \\
\text { Depresión; } \\
\text { Enfermería Peri } \\
\text { operatoria; } \\
\text { Cirugía } \\
\text { Torácica; } \\
\text { Complicaciones } \\
\text { Posoperatorias }\end{array}$ & 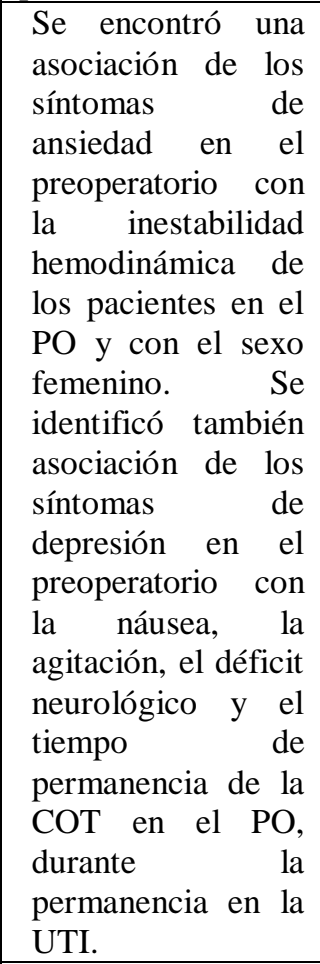 & $\begin{array}{l}\text { En este escenario, } \\
\text { los enfermeros } \\
\text { tienen un } \\
\text { importante papel, } \\
\text { ya que son los } \\
\text { principales } \\
\text { responsables de la } \\
\text { orientación de } \\
\text { enfermería, en } \\
\text { todo el } \\
\text { peri operatorio. }\end{array}$ \\
\hline $\begin{array}{l}\text { Dr. Frank Daniel } \\
\text { Martos Benítez, } \\
\text { Dra. Anarelys }\end{array}$ & $\begin{array}{r}\text { Google } \\
\text { académico }\end{array}$ & 2017 & $\begin{array}{l}\text { Cirugía } \\
\text { oncológica, } \\
\text { complicaciones } \\
\text { cardiovasculare }\end{array}$ & $\begin{array}{lr}\begin{array}{l}\text { Síndrome } \\
\text { agudo }\end{array} & \begin{array}{r}\text { coronario } \\
\text { tSCA); }\end{array} \\
\text { taquicardia } & \\
\text { ventricular } & (\mathrm{TV}) ;\end{array}$ & $\begin{array}{l}\text { No se r aplicó } \\
\text { ningún rol de } \\
\text { enfermería. }\end{array}$ \\
\hline
\end{tabular}




\begin{tabular}{|c|c|c|c|c|c|}
\hline $\begin{array}{l}\text { Gutiérrez } \\
\text { Noyola } \\
\text { Complicaciones } \\
\text { cardiovasculares } \\
\text { postoperatorias } \\
\text { en cirugía mayor } \\
\text { oncológica. }\end{array}$ & & & $\begin{array}{l}\mathrm{s}, \quad \text { paro } \\
\text { cardiorrespirato } \\
\text { rio, } \\
\text { insuficiencia } \\
\text { cardiaca aguda, } \\
\text { resultados } \\
\text { clínicos. }\end{array}$ & $\begin{array}{l}\text { taquicardias supra } \\
\text { ventriculares } \\
\text { (excepto taquicardia } \\
\text { sinusal); paro } \\
\text { cardiorrespiratorio } \\
\text { (PCR); } \\
\text { tromboembolismo } \\
\text { pulmonar (TEP); } \\
\text { insuficiencia } \\
\text { cardiaca aguda } \\
\text { (ICA); y crisis } \\
\text { hipertensiva } \\
\text { postoperatoria } \\
\text { (HTAP), definida } \\
\text { como presión arterial } \\
\geq 160 / 110 \text { mmHg } \\
20 .\end{array}$ & \\
\hline $\begin{array}{l}\text { Michelle Nacur } \\
\text { Lorentz, TSA, } \\
\text { Bruna Silviano } \\
\text { Brandão Vianna } \\
\text { Arritmias } \\
\text { Cardíacas y } \\
\text { Anestesia }\end{array}$ & Scielo & 2015 & $\begin{array}{l}\text { Anestesia; } \\
\text { enfermedades, } \\
\text { Cardíacas. }\end{array}$ & • Arritmias & $\begin{array}{l}\text { Se aplico el rol de } \\
\text { enfermería } \\
\text { investigativo ya } \\
\text { que el estudio fue } \\
\text { creado por un } \\
\text { grupo } \\
\text { enfermeras. }\end{array}$ \\
\hline 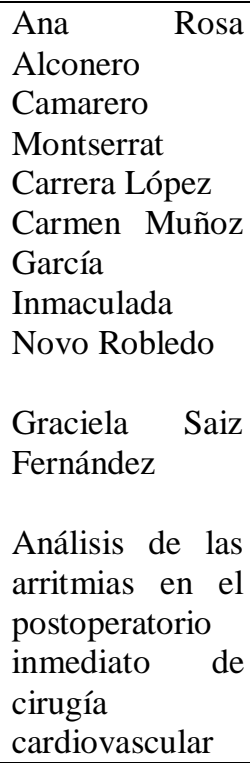 & $\begin{array}{l}\text { Revista } \\
\text { enfermería } \\
\text { intensiva }\end{array}$ & 2019 & $\begin{array}{l}\text { Enfermería. } \\
\text { Cirugía } \\
\text { cardiovascular. } \\
\text { Arritmias. } \\
\text { Postoperatorio }\end{array}$ & Las arritmias & $\begin{array}{l}\text { No se aplicó } \\
\text { ningún rol de } \\
\text { enfermería. }\end{array}$ \\
\hline $\begin{array}{l}\text { Fernando } \\
\text { Enríquez, } \\
\text { AntonioJiménez } \\
\text { Taquiarritmias } \\
\text { postoperatorias } \\
\text { en la cirugía } \\
\text { cardíaca }\end{array}$ & $\begin{array}{l}\text { sciencedire } \\
\mathrm{ct}\end{array}$ & 2015 & $\begin{array}{l}\text { Cardiopatías, } \\
\text { congénitas, } \\
\text { Arritmias, } \\
\text { Cirugía cardíaca }\end{array}$ & $\begin{array}{l}\text { La incidencia de } \\
\text { arritmias } \\
\text { postoperatorias } \\
\text { depende de la edad } \\
\text { del paciente, la } \\
\text { enfermedad de base, } \\
\text { el tratamiento } \\
\text { quirúrgico y las } \\
\text { diferentes prácticas } \\
\text { quirúrgicas locales. } \\
\text { En el periodo } \\
\text { postoperatorio } \\
\text { precoz se han } \\
\text { observado varios } \\
\text { factores de riesgo } \\
\text { como son la edad (a }\end{array}$ & $\begin{array}{l}\text { Se aplicó el rol de } \\
\text { enfermería } \\
\text { investigativo ya } \\
\text { que el estudio fue } \\
\text { creado por un } \\
\text { grupo } \\
\text { enfermeras. }\end{array}$ \\
\hline
\end{tabular}




\begin{tabular}{|l|l|l|}
\hline & & $\begin{array}{l}\text { menor edad, mayor } \\
\text { susceptibilidad), el } \\
\text { bajo peso, la mayor } \\
\text { duración de la CEC, } \\
\text { la mayor } \\
\text { complejidad y la } \\
\text { quirúrgica y de } \\
\text { presencia } \\
\text { defectos residuales. }\end{array}$ \\
\hline
\end{tabular}

\section{Análisis de los resultados}

De los 100 artículos que hemos encontrado luego de una ardua revisión bibliográfica, llegamos a la conclusión de que 20 de los artículos revisados son aquellos que mayor relevancia presentan con nuestro tema principal de nuestro proyecto integrador de saberes" PIS ", ya que el tema el cual hemos abordado es netamente complicaciones cardiovasculares posoperatorias, el cual va de la mano con el rol o los roles de enfermería, que el profesional ejecuta para restablecer la salud de los pacientes que presentan dichas complicaciones posquirúrgicas. El objetivo primordial del personal de enfermería es restablecer o mantener la salud de los pacientes en óptimas condiciones, dentro de la realización y ejecución del PAE ejerciendo sus roles no solo asistencial, sino investigativo ya que se debe estar en contaste búsqueda bibliográfica de las compilaciones posquirúrgicas que pueden sufrir los pacientes, además del rol educativo para logar que los pacientes postquirúrgicos estén en óptimas condiciones al momento del que el personal de enfermería se auto eduque a él y su familia sobre la complicación posquirúrgica que presenta. Al analizar los artículos antes mostrado, llegamos a la conclusión de que unas de las complicaciones más frecuentes que presentan los pacientes con complicaciones cardiovasculares post quirúrgicas son las arritmias e hipertensión arterial ya que son las que mayor relevancia poseen en la investigación seguidas, al estar en las bases de datos antes mencionadas (se consultaron diferentes bases de datos de carácter internacional y nacional; tales como Scielo, Cochrane, Bvs, Revicardiologia, Etc), una vez que empezamos a realizar la búsqueda bibliográfica haciendo énfasis en las palabras claves como :Complicaciones cardiovasculares, hipertensión, Complicaciones cardiovasculares, arritmia , Hipertensión arterial, complicación cardiovascular, Enfermería. Cirugía cardiovascular. Arritmias Postoperatorias, Cardiopatías, congénitas, Arritmias, Cirugía cardíaca, si bien al digitar las palabras claves se nos desplegó un aproximado de 100 artículos, de los cuales, dentro de la revisión bibliográfica exhaustiva, fuimos descartando artículos que no hacían referencia al tema principal de tratamos, quedando solamente 23 artículos que tienen más acercamiento con las complicaciones cardiovasculares posquirúrgicas, las cuales abordamos dentro del presente estudio bibliográfico.

\section{Conclusiones}

- De acuerdo a la revisión sistemática realizada se encontró que las principales complicaciones cardiovasculares en la etapa postoperatoria fueron: Taquicardias supraventriculares, paro cardiorrespiratorio y crisis hipertensiva postoperatoria, estas presentes en pacientes con falla cardiaca que han sido intervenidos de angioplastia.

- El rol de la enfermera que más desempeña es asistencial e investigativo porque desarrolla actividades que preservan la dignidad e integridad del paciente con el fin de mejorar la salud de este, realizando intervenciones de enfermería y respondiendo a sus necesidades.

Colaboradores en la Investigación: Chancay Saltos Jazmín Jamileth, Campuzano López Bryan Ariel, Intriago Mero Juliana Noemi, Mieles Macías María Lizbeth, Pinargote Moreira Gema Jazmín, Sornoza Almendariz Jennifer Estefanía, Vera Cedeño María Merced.

Estudiantes de la Carrera de Enfermería de la Universidad Estatal del Sur de Manabí.

\section{Bibliografía}

AE. Delgado Martína, J. S. (2003). Recuperado el 2020, de Cochrane : https://www.elsevier.es/es-revistamedicina-integral-63-articulo-manejo-crisis-hipertensivas-13045397 
Alondra Reyes. (29 de Marzo de 2017). Recuperado el 14 de Julio de 2020, de Geneally: https://view.genial.ly/58dc7619ec4cd60274c8c454/interactive-content-6-esferas-del-desarrollohumano\#: :text=6\%20esferas $\% 20 \mathrm{del} \% 20$ desarrollo $\% 20$ humano $\% 20$ by $\% 20$ alondra26reyes $\% 20$ on\%20Genially\&text=Bases\%20gen\%C3\%A9ticas\%20del\%20desarrollo\%2C\%20crecimiento,s alud

Anonimo. (s.f.). Recuperado el 19 de Julio de 2020, de FACTORES DE RIESGO CARDIOVASCULAR https://www.fmed.uba.ar/sites/default/files/201903/Factores\%20de\%20riesgo\%20cardiovascular.pdf

Asociación Española Contra el Cancer. (2018). La Vejiga Anatomía. AECC. Recuperado el 14 de Julio de 2020, de https://www.aecc.es/es/todo-sobre-cancer/tipos-cancer/cancervejiga/anatomia\#: :text=La\%20vejiga\%20forma\%20parte\%20del,a\%20trav\%C3\%A9s\%20de\% 20la\%20uretra.

B Bzoma, J Kostro, Un Hellmann, A Chamienia, S Hać, A Dębska-Ślizień, Z Śledziński. (2018). Recuperado el 10 de Agosto de 2020, de PudMed: https:/pubmed.ncbi.nlm.nih.gov/30056916/

Benitez, Noyola y Victores. (Marzo de 2016). Complicaciones postoperatorias y resultados clínicos en pacientes operados por cáncer torácico y gastrointestinal: Estudio de cohorte prospectivo. Revista Brasileira de Terapia Intensiva, 28(1).

Bertha S, Rodríguez A. (2015). Características del PAE. Recuperado el 14 de Julio de 2020, de Informacion sobre Enfermeria: https://www.congresohistoriaenfermeria2015.com/caracteristicas.html

Burgués, S. (2017). Obtenido de http://www.scielo.edu.uy/scielo.php?script=sci_arttext\&pid=S1688-04202017000300370

Carlos Eduardo Hernández García, Diana Chaparro. Gustavo Ramos,José de la Hoz. (2018). Nefrolitotomía percutánea ambulatoria: serie. Urol. colomb.

Carlos, S. (2018). Recuperado el 18 de 07 de 2020, de www.fbbva.es: https://www.fbbva.es/microsites/salud_cardio/mult/fbbva_libroCorazon_cap69.pdf

Carol R Wade, K. K. (2004). Recuperado el 08 de Septiembre de 2020, de pubmed: https://pubmed.ncbi.nlm.nih.gov/14974522/

Cátedra de cirugía. (8 de Agosto de 2011). Postoperatorio. Universidad Nacional de Córdova. Recuperado el 10 de Julio de 2020, de https://blogs.unc.edu.ar/cirugia/2011/08/08/postoperatorio/

cirugiacardiovascular. (2019). Recuperado el 18 de Agosto de 2020, de cirugiacardiovascular.co: https://cirugiacardiovascular.co/alimentacion-despues-de-una-cirugia-cardiovascular/

codem. (2018). Recuperado el 11 de Agosto de 2020, de www.codem.es: http://www.codem.es/Adjuntos/CODEM/Documentos/Informaciones/Publico/9e8140e2-cec74df7-8af9-8843320f05ea/8c06b7e5-ca29-40c6-ab63-f84959a87362/c618e862-974d-4faf-809366eae984e3da/TRABAJO_CONGRESO_GRAFICA_AJUSTADA.pdf

Complicaciones Renales. (Junio de 2016). Bd. Recuperado el 9 de Julio de 2020, de https://www.bd.com/eses/our-products/diabetes-care/diabetes-learning-center/about-diabetes/complications-caused-bydiabetes/kidney-complications

Cuidateplus. (2017). Recuperado el 18 de Agosto de 2020, de cuidateplus.marca.com: https://cuidateplus.marca.com/enfermedades/respiratorias/embolia-pulmonar.html

Cunha, Dos Santos , Costa, Arimatea, Russo y Oliveira. (Abril de 2017). Prevalencia de infección del tracto urinario en el primer mes posterior al trasplante renal en un hospital universitario. Revista de enfermagem Uerj Nursing Journal, 25.

D. Cáceres, K. M.-S. (2016). Obtenido de Scielo: http://www.scielo.org.co/scielo.php?script=sci_abstract\&pid=S0120$56332016000500420 \& \operatorname{lng}=\mathrm{en} \& n \mathrm{~mm}=\mathrm{iso} \& \operatorname{lng}=\mathrm{es}$

Elsa Solá, Pere Ginés. (27 de Abril de 2018). Síndrome Hepatorrenal. Sociedad Española de Nefrología. Recuperado el 15 de Julio de 2020, de https://www.nefrologiaaldia.org/es-articulo-sindromehepatorrenal-161

ELSEVIER. (Abril-Junio de 2015). Incidencia de la infección urinaria en pacientes postoperados de cirugía cardiaca: estudio comparativo según el dispositivo de sondaje. ELSEVIER, 26(2). Recuperado el 15 de Julio de 2020, de https:/www.elsevier.es/es-revista-enfermeria-intensiva-142-articuloincidencia-infeccion-urinaria-pacientes-postoperados-S1130239914000959

elsevier. (2018). Recuperado el 11 de Agosto de 2020, de www.elsevier.es: https://www.elsevier.es/esrevista-medicina-integral-63-articulo-manejo-crisis-hipertensivas13045397\#: :text=Crisis\%20hipertensiva.,Emergencia\%20hipertensiva.

Emilia Pawłowska-Krajka, Adam Dorobek. (2017). Recuperado el 10 de Agosto de 2020, de PudMed: https://pubmed.ncbi.nlm.nih.gov/29410894/

Emily A Kidd; Fiona Stewart; Nadine C Kassis; Emily Hom. (2015). Recuperado el 17 de Agosto de 2020, de 
https://www.cochranelibrary.com/cdsr/doi/10.1002/14651858.CD004203.pub3/full/es?highlight Abstract=vias\%7Curetral\%7Cintermitent\%7Cpermanent\%7Curetrales\%7Cintermitentes\%7Cper manentes\%7Ci\%7Co

Emily R ThompsonSarah A HosgoodMichael L NicholsonColin H Wilson. (29 de January de 2018) Extracción temprana versus tardía del stent ureteral después del trasplante de riñón. Recuperado el 15 de Agosto de 2020, de Cochrane Library Reviews: https://www.cochranelibrary.com/cdsr/doi/10.1002/14651858.CD011455.pub2/full/es?highlight Abstract=renal\% 7Ccomplicaciones\% 7Cpostoperatorias\% 7Cpostoperatoria\% 7Ccomplicacion\% 7 Cpostoperatori\% 7Crenales

Empendium. (2018). Recuperado el 18 de Agosto de 2020, de empendium.com: https://empendium.com/manualmibe/chapter/B34.II.2.19.2.

encolombia. (2020). Recuperado el 11 de Agosto de 2020, de encolombia.com: https://encolombia.com/medicina/revistas-medicas/enfermeria/ve-

114/arritmiasmasfrecuentesenelpostoperatorioinmediato1/

Fabricio, S. (2017). Recuperado el 18 de 07 de 2020, de www.revista-portalesmedicos.com: https://www.revista-portalesmedicos.com/revista-medica/cuidados-de-enfermeria-insuficienciacardiaca/

fundaciondelcorazon. (2019). Recuperado el 11 de Agosto de 2020, de fundaciondelcorazon.com: https://fundaciondelcorazon.com/informacion-para-pacientes/enfermedadescardiovasculares/tromboembolismo-pulmonar.html

fundacionindex. (2019). Recuperado el 11 de Agosto de 2020, de www.fundacionindex.com: http://www.fundacionindex.com/gomeres/?p=626

G Orlandova, L. B. (2020). Recuperado el 08 de 09 de 2020, de Scielo: http://scielo.sld.cu/scielo.php?script=sci_arttext\&pid=S2218-

36202020000200293\&lng=pt\&nrm=iso\&tlng=pthttp://scielo.sld.cu/scielo.php?script=sci_arttext \&pid=S2218-

36202020000200293\&lng=pt\&nrm=iso\&tlng=pthttp://scielo.sld.cu/scielo.php?script=sci_arttext \&pi

Geosalud. (2018). Recuperado el 18 de Agosto de 2020, de www.geosalud.com: https://www.geosalud.com/hipertension/crisishipertensiva.htm\#: :text=La\%20droga\%20de\%20e lecci\%C3\%B3n\%20para,e\%20impredecible\%20est\%C3\%A1n\%20totalmente\%20contraindicad os $\% 20$.

González, Hernández. (enero-abril de 2018). Complicaciones anestésicas intraoperatorias y posoperatorias de la nefrolitotomía percutánea en pacientes con litiasis coraliforme. scielo.

Healthwise. (11 de Agoato de 2019). Filtración glomerular (GFR). Cigna. Recuperado el 15 de Julio de 2020, de https://www.cigna.com/individuals-families/health-wellness/hw-en-espanol/temas-desalud/filtracion-glomerular-aa154102

imss.mx. (2017). Recuperado el 18 de Agosto de 2020, de www.imss.gob.mx: http://www.imss.gob.mx/sites/all/statics/guiasclinicas/591GRR.pdf

orge Olmos Salamanca, P. V. (2016). Recuperado el 18 de 07 de 2020, de www.sciencedirect.com: https://www.sciencedirect.com/science/article/pii/S012173721600011X

Jorge Olmos Salamanca, P. V. (2016). Recuperado el 18 de 07 de 2020, de www.sciencedirect.com: https://www.sciencedirect.com/science/article/pii/S012173721600011X

Juan Sebastián Barco López, A. d. (2017). Recuperado el 03 de Septiembre de 2020, de www.icpcolombia.org: http://www.icpcolombia.org/dev/wpcontent/uploads/2016/08/2008.04.21-Las-esferas-del-desarrollo.pdf

Julia Máxima Uriarte. (Diciembre de 2019). Sistema excretor. Características.com. Recuperado el 14 de Julio de 2020, de https://www.caracteristicas.co/sistema-excretor/

Laura Estefania Alvarado Robles, Dayra Gisella Angulo DE León. (2013-2014). IMPACTO DEL ROL DE ENFERMERIA EN PACIENTES CON INSUFICIENCIA RENAL CRÓNICA. Tesis de grado previo a la obtención del titúlo de Enfermera, Guayaquil. Recuperado el 10 de Julio de 2020, de http://repositorio.ug.edu.ec/bitstream/redug/8728/1/TESIS\%20FINAL.pdf

Laura Estefania Alvarado Robles, Dayra Gisella Angulo de León. (2014-2015). Impacto del Rol de enfermeria en pacientes con complicaciones renales. Tesis de Licenciatura en Enfermería, Universidad de Guayaquil, Guayaquil. Recuperado el 19 de Agosto de 2020, de http://repositorio.ug.edu.ec/bitstream/redug/8728/1/TESIS\%20FINAL.pdf

LEONARDO, A. M. (2017). Recuperado el 19 de Julio de 2020, de RIESGO DE ENFERMEDAD CARDIOVASCULAR: http://dspace.uniandes.edu.ec/bitstream/123456789/6881/1/PIUAMED078-2017.pdf 
M Hernández, P. G., Monte Bautista, C. V., \& Valdéz Becerril, G. A. (2016). Obtenido de Scielo: http://www.scielo.org.mx/scielo.php?script=sci_arttext\&pid=S0301-696X2018000300231

Manual MSD. (Febrero de 2015). Traumatismos vesicales. MSD. Recuperado el 15 de Julio de 2020, de https://www.msdmanuals.com/es-ec/professional/lesiones-y-envenenamientos/traumatismos-delaparato-urogenital/traumatismos-vesicales

Manual MSD. (Agosto de 2016). Lesiones ureterales. MSD. Recuperado el 15 de Julio de 2020, de https://www.msdmanuals.com/es-ec/hogar/traumatismos-y-envenenamientos/lesiones-de-lasv\%C3\%ADas-urinarias-y-de-los-genitales/lesiones-ureterales

Mañero, D. M. (s.f.). Recuperado el 19 de Julio de 2020, de FIBRILACIÓN AURICULAR: Dr. Moisés Rodríguez Mañero

Mañero, D. M. (s.f.). Recuperado el 19 de Julio de 2020, de MUERTE SÚBITA: https://fundaciondelcorazon.com/informacion-para-pacientes/enfermedadescardiovasculares/muerte-subita.html

Mañero, D. M. (s.f.). Recuperado el 19 de Julio de 2020, de SÍNDROME DE BRUGADA: https://fundaciondelcorazon.com/informacion-para-pacientes/enfermedadescardiovasculares/sindrome-de-brugada.html

María C Sánchez. (2014). Prevención, diagnóstico y tratamiento de la enfermedad Renal. Recuperado el 10 de Julio de 2020, de Universidad del Azuay: http://dspace.uazuay.edu.ec/bitstream/datos/3940/1/10544.pdf

Mariano A. Núñez, Bernardo Chiconi, Gonzalo Linares, Federico Aveni, Mauricio Pilot, Germán Albino. (2018). Litiasis renal: ¿Puede el abordaje percutáneo resolver todos los casos? Rev. argent. urol. (1990).

Maricela Torres Esperón, Caridad Dandicourt Thomas y Aida Rodríguez Cabrera. (Mayo - Agosto de 2005). Funciones de enfermería en la atención primaria de salud. Scielo, 21(3-4). Recuperado el 14 de Julio de 2020, de Scielo: http://scielo.sld.cu/scielo.php?script=sci_arttext\&pid=S086421252005000300007

Maricela Torres Esperón, Caridad Dandicourt Thomas, Aida Rodríguez Cabrera. (21 de Mayo de 2029). Funciones o Roles de la Enfermera. Yo amo Enfermería. Recuperado el 10 de Julio de 2020, de https://yoamoenfermeriablog.com/2019/05/21/funciones-o-roles-de-enfermeria/

Martinez, J. (2017). Recuperado el 18 de Agosto de 2020, de es.slideshare.net: https://es.slideshare.net/Juliiom13/complicaciones-postoperatorias-47204958

Mayo Clinic. (19 de Marzo de 2019). Infección de las vías urinarias. Infección de las vías urinarias. Recuperado el 15 de Julio de 2020, de https://www.mayoclinic.org/es-es/diseasesconditions/urinary-tract-infection/symptoms-causes/syc-20353447

Mayoclinic. (2017). Recuperado el 18 de Agosto de 2020, de www.mayoclinic.org: https://www.mayoclinic.org/es-es/diseases-conditions/sudden-cardiac-arrest/symptomscauses/syc-20350634

Mayoclinic. (2018). Recuperado el 18 de Agosto de 2020, de www.mayoclinic.org: https://www.mayoclinic.org/es-es/diseases-conditions/ventricular-tachycardia/symptomscauses/syc-20355138

Mayoclinic. (2019). Recuperado el 18 de Agosto de 2020, de www.mayoclinic.org: https://www.mayoclinic.org/es-es/diseases-conditions/supraventricular-tachycardia/symptomscauses/syc-20355243

medigraphic. (2018). Recuperado el 11 de AGOSTO de 2020, de www.medigraphic.com: https://www.medigraphic.com/pdfs/medcri/ti-2013/ti133f.pdf

medlineplus. (2019). Recuperado el 11 de Agosto de 2020, de medlineplus.gov: https://medlineplus.gov/spanish/ency/article/007639.htm

medwave. (2018). Recuperado el 11 de AGOSTO de 2020, de www.medwave.cl: https://www.medwave.cl/link.cgi/Medwave/Reuniones/Anestesiologia/Octubre/2438

Michael Heung, Jay L Koyner. (2015). Recuperado el 12 de Agosto de 2020, de PubMed: https://pubmed.ncbi.nlm.nih.gov/25795497/

Muñoz, D. A. (s.f.). Recuperado el 19 de Julio de 2020, de TROMBOEMBOLISMO PULMONAR: https://fundaciondelcorazon.com/informacion-para-pacientes/enfermedadescardiovasculares/tromboembolismo-pulmonar.html

Muñoz, D. A. (s.f.). Recuperado el 19 de Julio de 2020, de SÍNDROME DE TAKO-TSUBO: https://fundaciondelcorazon.com/informacion-para-pacientes/enfermedadescardiovasculares/sindrome-de-tako-tsubo.html

Muñoz, D. A. (s.f.). Recuperado el 19 de Julio de 2020, de COARTACIÓN DE AORTA: https://fundaciondelcorazon.com/informacion-para-pacientes/enfermedadescardiovasculares/coartacion-de-aorta.html 
Muñoz, D. A. (s.f.). Recuperado el 19 de Julio de 2020, de FORAMEN OVAL PERMEABLE: https://fundaciondelcorazon.com/informacion-para-pacientes/enfermedadescardiovasculares/foramen-oval-permeable.html

Muñoz, D. A. (s.f.). Recuperado el 19 de Julio de 2020, de SíNDROME DE MARFAN: https://fundaciondelcorazon.com/informacion-para-pacientes/enfermedadescardiovasculares/sindrome-de-marfan.html

Muñoz, D. A. (s.f.). Recuperado el 2020 de Julio de 2020, de DUCTUS ARTERIOSO: https://fundaciondelcorazon.com/informacion-para-pacientes/enfermedadescardiovasculares/ductus-arterioso-persistente.html

Muñoz, D. A. (s.f.). Recuperado el 19 de Julio de 2020, de TRANSPOSICIÓN DE GRANDES VASOS: https://fundaciondelcorazon.com/informacion-para-pacientes/enfermedadescardiovasculares/transposicion-de-grandes-vasos.html

my-ekg. (2018). Recuperado el 11 de Agosto de 2020, de www.my-ekg.com: https://www.myekg.com/arritmias-cardiacas/taquicardias-ventriculares/taquicardiasventriculares.html\#: :text=Taquicardia\%20Ventricular\&text=Las\%20taquicardias\%20ventricula res\%20(TV)\%20son,con\%20una\%20frecuencia\%20cardiaca\%20elevada.

my-ekg. (2019). Recuperado el 11 de Agosto de 2020, de www.my-ekg.com: https://www.myekg.com/arritmias-cardiacas/taquicardias-supraventriculares.html

N Abdo, T Murez, L Cabaniols, M Robert, S Marchal, N Amadane, S Thezens, F Iborra, R Thuret. (2019). Resultados de las revisiones quirurgicas por complicaciones ureterales despues del transplante renal. Recuperado el 11 de Agosto de 2020, de PubMed: https://pubmed.ncbi.nlm.nih.gov/31400962/

Nahum Montahud Rubio. (Marzo de 2017). Sistema excretor: características, partes y funcionamiento. Psicología y mente. Recuperado el 16 de Julio de 2020, de https://psicologiaymente.com/salud/sistema-

excretor\#: :text=E1\%20sistema\%20excretor\%2C\%20tambi\%C3\%A9n\%20denominado,aprovec hables\%20por\%20el\%20cuerpo\%20humano.

Nancy Burns, S. K. (2012). Investigación en Enfermería (5ta ed.). (Elsevier, Ed.) Barcelona (España): Edide, S.L.

National Institute of Diabetes and Digestive and Kidney Diseases. (Junio de 2018). Los riñones y su funcionamiento. NIH. Recuperado el 15 de Julio de 2020, de https://www.niddk.nih.gov/healthinformation/informacion-de-la-salud/enfermedades-rinones/rinones-

funcionamiento\#: :text=Los $\% 20$ ri\%C3\%B1ones\%20son $\% 20 \mathrm{dos} \% 20 \% \mathrm{C} 3 \%$ B3rganos,lado\%20 de\%20la\%20columna\%20vertebral.

Noemi BA Roy; Patricia M Fortin; Katherine R Bull; Carolyn Doree; Marialena Trivella; Sally Hopewell. (2017). Recuperado el 15 de Agosto de 2020, de Cochrane Systematic Review: https://www.cochranelibrary.com/cdsr/doi/10.1002/14651858.CD012380.pub2/full/es?highlight Abstract=con\%7Cdisminuir\%7Cen\%7Cparas\%7Cintervencion\%7Crenales\%7Co\%7Crenal\% 7C pacient \%7Ccomplicaciones\%7Cprevenir\%7Cpara\%7Cdrepanocitosi\%7Cdrepanocitosis\%7Cpac ientes $\% 7$

Omar Alkandari, Lieuko, Diane Hebert, Valerie Langlois, Natasha A Jawa, Rulan S Parekh, Lisa A Robison. (2018). Lesion renal aguda en niños con trasplante renal. Recuperado el 11 de Agosto de 2020, de PubMed: https://pubmed.ncbi.nlm.nih.gov/30242029/

OMS. (2018). Recuperado el 11 de AGOSTO de 2020, de MEDICALCENTER: https://apps.who.int/iris/bitstream/handle/10665/44233/9789243598598_spa.pdf;jsessionid=FFD 66490049ED776E643E8D572F74489?sequence=1

Oneys del Carmen De Arco- Canoles, Zuleima Karina Suarez-Calle. (13 de Abril de 2018). Rol de los profesionales de enfermría en el sistema de salud Colombiano. Recuperado el 10 de Julio de 2020, de http://sired.udenar.edu.co/4536/1/Rol\%20de\%20los\%20prof.pdf

Owoicho Adogwa, Aladine A Elsamadicy, Amanda Sergesketter, Deborah Oyeyemi, Diego Galan, Victoria D Vuong, Syed Khalid, Joseph Cheng, Carlos A Bagley, Isaac O Karikari. (2017). Recuperado el 15 de Agosto de 2020, de PubMed: https://pubmed.ncbi.nlm.nih.gov/29109065/

Pereira, Badaoui, Dantas, Sanches, Alvares, Scaranello, Castro, Silva y Laranja. (2016). Lesão renal aguda no pós-operatório de cirurgias não cardíacas em pacientes com recuperação na unidade de terapia intensiva. Revista Sociedade Brasileira de Clínica Médica, 14(4).

Pérez, D. P. (s.f.). Recuperado el 19 de Julio de 2020, de INFARTO DE MIOCARDIO: https://fundaciondelcorazon.com/informacion-para-pacientes/enfermedades-

cardiovasculares/infarto.html 
Priscila Gonzáles Salcedo; Alida Mireya Chaves Reyes,. (Julio de 2009). Proceso de atención de enfermería desde la perspectiva docente. Redalyc, 11(2). Recuperado el 14 de Julio de 2020, de https://www.redalyc.org/pdf/1452/145220480004.pdf

Regi Freeman, E. K. (2016). Recuperado el 08 de Septiembre de 2020, de pubmed: https://pubmed.ncbi.nlm.nih.gov/27254638/

revcardiologia. (2019). Recuperado el 11 de Agosto de 2020, de www.www.revcardiologia.sld.cu.sld.cu: http://www.revcardiologia.sld.cu/index.php/revcardiologia/article/view/633/html_36

revespcardiol. (2018). Recuperado el 11 de Agosto de 2020, de www.revespcardiol.org: https://www.revespcardiol.org/es-insuficiencia-cardiaca-aguda-epidemiologia-factores-articuloS0300893214006563\#: :text=La\%20insuficiencia\%20cardiaca\%20aguda\%20(ICA,hospitalizaci $\%$ C3\%B3n\%20del\%20paciente1\%E2\%80\%933.

Reyes, A. (2017). Recuperado el 03 de Septiembre de 2020, de Genially : https://view.genial.ly/58dc7619ec4cd60274c8c454/interactive-content-6-esferas-del-desarrollo-humano. 